\title{
A atenção primária à saúde na coordenação das redes de atenção: uma revisão integrativa
}

\author{
Primary Health Care in the coordination of health care networks: \\ an integrative review
}

Ludmila Barbosa Bandeira Rodrigues ${ }^{1}$

Patricia Costa dos Santos Silva ${ }^{1}$

Rarianne Carvalho Peruhype ${ }^{1}$

Pedro Fredemir Palha ${ }^{1}$

Marcela Paschoal Popolin ${ }^{1}$

Juliane de Almeida Crispim ${ }^{1}$

Ione Carvalho Pinto ${ }^{1}$

Aline Aparecida Monroe ${ }^{1}$

Ricardo Alexandre Arcêncio ${ }^{1}$

${ }^{1}$ Escola de Enfermagem de Ribeirão Preto,

Universidade de São Paulo. Av. Bandeirantes 3900,

Monte Alegre. 14.040-902 Ribeirão Preto SP Brasil. ludbbr@yahoo.com.br
Abstract Health systems organized in health care networks and coordinated by Primary Health Care can contribute to an improvement in clinical quality with a positive impact on health outcomes and user satisfaction (by improving access and resolubility) and a reduction in the costs of local health systems. Thus, the scope of this paper is to analyze the scientific output about the evidence, potential, challenges and prospects of Primary Health Care in the coordination of Health Care Networks. To achieve this, the integrative review method was selected covering the period between 2000 and 2011. The databases selected were Medline (Medical Literature Analysis and Retrieval System online), Lilacs (Latin American Literature in Health Sciences) and SciELO (Scientific Electronic Library Online). Eighteen articles fulfilled the selection criteria. It was seen that the potential impacts of primary care services supersede the inherent weaknesses. However, the results revealed the need for research with a higher level of classification of the scientific evidence about the role of Primary Healh Care in the coordination of Health Care Networks.

Key words Primary health care, Intersectoral action, Health care networks, Coordination of care
Resumo Os sistemas de saúde, organizados em Redes de Atenção e coordenados pela Atenção Primária à Saúde, podem contribuir para a qualidade clínica, com resultados sanitários de impacto positivo, na satisfação dos usuários (pela melhoria do acesso e resolubilidade) e na redução dos gastos dos sistemas locais de saúde. Dessa forma, propôs-se analisar a produção científica acerca das evidências, potencialidades, desafios e perspectivas da Atenção Primária à Saúde na coordenação das Redes de Atenção. Para atingir o objetivo, definiu-se como método a revisão integrativa, considerando o período temporal de 2000 a 2011. As bases de dados selecionadas foram Medline (Medical Literature Analysis and Retrieval Sistem on-line), Lilacs (Literatura Latino-Americana em Ciências de Saúde) e SciELO (Scientific Electronic Library Online). Atenderam aos critérios de seleção 18 artigos. Pode-se observar que as potencialidades acerca dos serviços de Atenção Primária se sobressaíram às fragilidades. Contudo, destaca-se a necessidade de pesquisas com maior nível de classificação das evidências científicas sobre a atuação da Atenção Primária à Saúde na coordenação das Redes de Atenção.

Palavras-chave Atenção primária à saúde, Ação intersetorial, Redes de atenção à saúde, Coordenação dos cuidados 


\section{Introdução}

As doenças crônicas não transmissíveis constituem hoje o maior desafio para os sistemas de saúde por modificarem o padrão de necessidades da população, o que lhes impõe uma configuração sensível à produção da equidade e com capacidade de resolubilidade dos problemas, não mais na perspectiva de cura, mas do cuidado. Dados da Organização Mundial da Saúde (OMS) revelam que as doenças crônicas não transmissíveis responderam em 2011 por $59 \%$ da mortalidade total do mundo, havendo uma estimativa sobressair os $78 \%$ já no ano de $2020^{1}$. Assim, vem sendo vislumbradas novas formas de integração dos sistemas de saúde para o enfrentamento desta nova realidade social, sendo uma delas inspiradas na Atenção Primária à Saúde $(\mathrm{APS})^{2}$, especialmente por meio do fortalecimento do atributo coordenação. Desde a década de 1990 um movimento por reformas dos sistemas de saúde tem se dado em todo mundo, para que respondam de forma mais resolutiva, corresponsável e justa às demandas sociais ${ }^{3,4}$.

Vale mencionar que o conceito de APS foi cunhado em 1920 pelo relatório Dawson, que os mencionava como o foco central do processo de regionalização no Reino Unido 5 . Todavia, a APS foi consagrada a partir da Conferência Internacional no ano de 1978, em Alma-Ata ${ }^{6}$, definida na ocasião como a porta de entrada dos sistemas de saúde e o primeiro elemento de um processo contínuo de atenção ${ }^{4}$.

A partir da conferência, as concepções e as práticas da APS se deram de forma diversificada em todo mundo, sendo que nos países periféricos foi ressonante um tom mais seletivo, passando a designar um pacote de intervenções de baixo custo para controle de determinados agravos, providas por pessoal de baixa qualificação profissional e sem a possibilidade de referência de nível de atenção de maior densidade tecnológica $^{7,8}$. Essa concepção foi fortemente fomentada pelos organismos internacionais, levando a APS a assumir um polo muito mais tecnocrático, com práticas racionalizadas, restrita à cesta de serviços, sendo colocada numa posição antagônica ao Estado de Direito.

Uma mobilização em âmbito nacional vem ocorrendo para que esta visão seja superada, colocando a APS como um eixo estruturante de um sistema, o que alguns autores definem como abrangente ou ampliada ${ }^{7-10}$. Estudo mostra que esta concepção está assentada no modelo de proteção social à saúde adotado em cada país ${ }^{11}$, e nestas localidades o eixo coordenação torna-se a insígnia dos sistemas de saúde inspirados na APS.

No artigo, assume-se coordenação como a capacidade de garantir a continuidade da atenção, de um modo integrado com os diferentes pontos de atenção ${ }^{9}$ e compreendida nas dimensões vertical, entre a APS e os demais níveis do sistema; e horizontal, que envolveria a articulação entre a própria equipe de APS, serviços de saúde e equipamentos sociais ${ }^{4}$.

No entanto, são grandes os desafios para que a APS assuma o papel de coordenadora de uma Rede de Atenção à Saúde (RAS). Dentre os nóscríticos apontados pela literatura, destacam-se a falta de política institucional direcionada ao fortalecimento da APS, a representação social das comunidades sobre este ponto de atenção, a falta de legitimidade social, a visão restrita de gestores que tendem a compreendê-la, por vezes, como seletiva, além da insuficiência de recursos qualificados para um modus faciendi desta estratégia, o que resulta numa notável carência de dispositivos de apoio e logística que favoreçam a inovação das práticas, o acolhimento dos usuários e a vinculação dos mesmos na $\mathrm{APS}^{10,11}$.

A coordenação das RAS pela APS implica que ela assuma papel central e estratégico de reordenamento do sistema de saúde, orientando o cuidado ao longo de todos os pontos de atenção e de toda a vida de uma comunidade ${ }^{12}$. Entende-se por RAS, organizações poliárquicas de conjuntos de serviços de saúde vinculadas entre si por uma missão única, por objetivos comuns e por uma ação cooperativa e interdependente que permite ofertar uma atenção contínua e integral à determinada população ${ }^{13}$.

Estudos evidenciam que a APS eleva sua capacidade de resolver os problemas de saúde por meio da coordenação das RAS, tornando os sistemas mais custo-efetivos, tanto em termos de organização interna, na alocação de seus recursos e na gestão clínica, como também pela sensibilidade de se ajustar de acordo com a conjuntura socioeconômica, demográfica e epidemiológi$\mathrm{ca}^{14,15}$. No campo das políticas públicas, a organização do sistema em redes possibilita a construção de vínculos de solidariedade e cooperação entre trabalhadores, serviços e comunidade ${ }^{16}$.

Há evidências de que o desenvolvimento da RAS tem se firmado como estratégia para a organização e a reestruturação do sistema de saúde, resultando em qualidade e impacto na produção dos serviços prestados à população ${ }^{14}$. No entanto, apesar de defesas favoráveis à APS, questionam-se quais as evidências científicas da sua 
efetividade na coordenação das RAS, as suas potencialidades e os desafios para que se conformem como coordenadora de uma rede progressiva de cuidados.

\section{Método}

A revisão integrativa da literatura foi adotada como método de agrupamento dos dados e síntese do conhecimento acerca da temática proposta, de modo a responder a seguinte questão norteadora: Quais as evidências científicas acerca das fragilidades, potencialidades, desafios e perspectivas da atenção primária à saúde na coordenação das redes de atenção?

Concernentes às definições de Cooper ${ }^{17}$ foram cumpridas as seguintes etapas: formulação do problema, levantamento de dados, avaliação dos dados coletados, análise e interpretação dos dados e apresentação dos resultados.

Conforme recomendações de Ganong ${ }^{18}$ e Broome $^{19}$ foram definidos os critérios de inclusão e exclusão adotados para a seleção dos manuscritos. Dessa forma, foram incluídos artigos que discorressem sobre a APS na coordenação das RAS, sendo excluídas dissertações, teses e notas editoriais. Optou-se por selecionar estudos publicados no período temporal de 2000 a 2011, escritos nos idiomas inglês, espanhol e português.

Medline (Medical Literature Analysis and Retrieval Sistem on-line), Lilacs (Literatura LatinoAmericana em Ciências de Saúde) e SciELO (Scientific Electronic Library Online) foram às bases de dados selecionadas para a realização da busca literária. Torna-se válido destacar que esta busca realizou-se de dezembro de 2011 a janeiro de 2012 de forma concomitante nas três.

Foram consideradas as seguintes palavraschave (empregadas de forma livre numa busca simples): redes de atenção à saúde (health care networks) e coordenação dos cuidados (coordination of care). Realizou-se, ainda, o cruzamento dos descritores controlados "atenção primária à saúde" e "ação intersetorial”, referenciados pelo Decs/Mesh. Os manuscritos foram selecionados pelo título e resumo, sendo, posteriormente, avaliados na íntegra para uma completa apreciação do material retido.

Os autores consideraram o instrumento proposto por Ursi ${ }^{20}$, adaptado pelo Grupo de Investigação em Reabilitação e Qualidade de Vida (GIRQ) da Escola de Enfermagem de Ribeirão Preto, da Universidade de São Paulo, para a extração de dados dos artigos. A identificação do estudo, as características metodológicas, a avaliação do rigor metodológico, as intervenções mensuradas e os resultados encontrados são algumas das informações exigidas pelo instrumento.

Os estudos investigados foram classificados por meio dos conceitos apresentados por Polit et al. ${ }^{21}$. A análise da qualidade do nível de evidência dos artigos selecionados foi realizada com base na classificação hierárquica de evidências científicas, proposta por Dawson ${ }^{22}$ com referência a Sackett et $\mathrm{al}^{23}$, estando os resultados apresentados de forma descritiva.

\section{Resultados}

Na primeira fase do estudo foram encontrados 1033 artigos, após a análise deste material, verificou-se que 18 manuscritos satisfaziam os critérios estabelecidos, conformando, portanto, a amostra final do estudo (Figura 1).

No Lilacs foram obtidos 405 artigos, dos quais 23 foram selecionados conforme o critério de inclusão e 382 excluídos. Já no Medline, foram

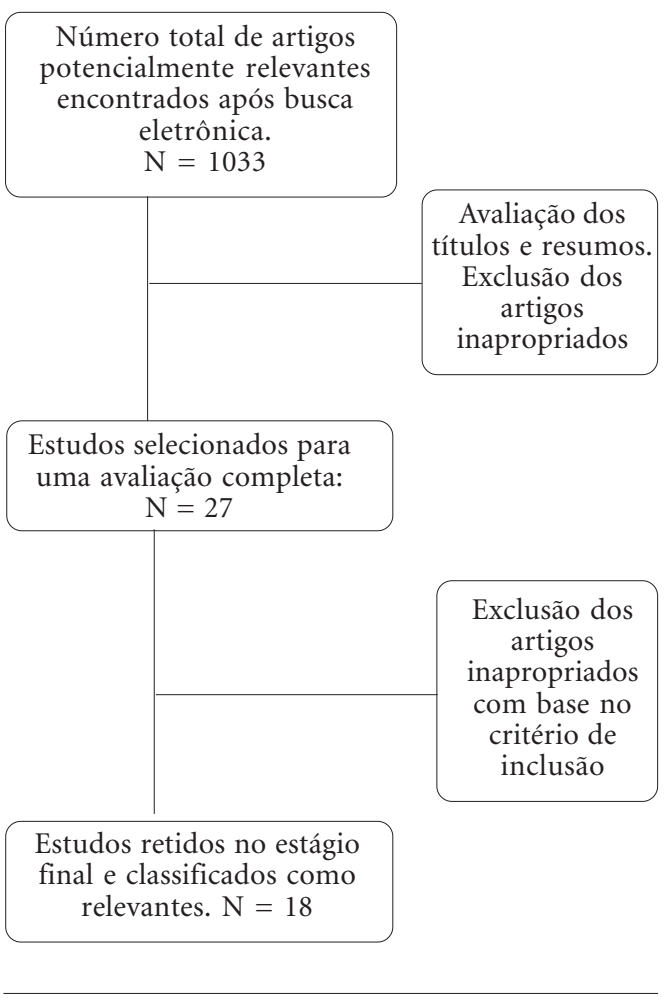

Figura 1. Fluxograma relacionado ao processo de seleção dos artigos. 
obtidos 628 artigos na totalidade, dos quais 4 foram selecionados e 624 excluídos; e no SciELO não foi encontrado nenhum artigo. Na fase final, 27 artigos foram selecionados para a análise completa do conteúdo e 18 destes retidos e incluídos na revisão integrativa. O Quadro 1 apresenta um breve resumo destes 18 estudos.
Após análise do material bibliográfico, constatou-se que $89 \%$ (16) eram estudos realizados no Brasil, 5,5\% (1) na Argentina e 5,5\% (1) na região das Américas. Quanto à linguagem empregada nos trabalhos, $15(83,4 \%)$ no idioma português, $11,1 \%$ (2) em espanhol e $5,5 \%$ (1) em inglês.

Quadro 1. Resumo dos artigos analisados na revisão integrativa, 2012.

\begin{tabular}{|c|c|c|c|c|}
\hline Referência & Objetivo & $\begin{array}{l}\text { Tipo de } \\
\text { estudo }\end{array}$ & $\begin{array}{c}\text { Plano } \\
\text { amostral }\end{array}$ & $\begin{array}{l}\text { Nível de } \\
\text { evidência }\end{array}$ \\
\hline $\begin{array}{c}\text { Giovanella, } \\
2011\end{array}$ & $\begin{array}{l}\text { Descrever e analisar as recentes mudanças } \\
\text { organizacionais no setor ambulatorial na } \\
\text { Alemanha que visaram à maior integração do } \\
\text { sistema de saúde e coordenação de cuidados }\end{array}$ & $\begin{array}{l}\text { Revisão da } \\
\text { literatura }\end{array}$ & Não se aplica & 4 \\
\hline $\begin{array}{c}\text { Almeida et al., } \\
2011\end{array}$ & $\begin{array}{l}\text { Descrever e analisar ações empreendidas em } \\
\text { quatro centros urbanos para fortalecer a } \\
\text { estratégia saúde da família (ESF) no Brasil }\end{array}$ & $\begin{array}{l}\text { Estudo de } \\
\text { caso }\end{array}$ & Conveniência & 4 \\
\hline $\begin{array}{c}\text { Sisson et al., } \\
2011\end{array}$ & $\begin{array}{l}\text { Examinar a implementação da Estratégia de } \\
\text { Saúde da Família e analisar suas possibilidades } \\
\text { em conduzir a orga-nização do sistema de saúde } \\
\text { quanto à integração à rede de serviços com } \\
\text { coordenação dos cuidados, desde a perspectiva } \\
\text { das famílias cadastradas, dos profissionais de } \\
\text { saúde e dos gestores }\end{array}$ & $\begin{array}{l}\text { Estudo de } \\
\text { caso }\end{array}$ & Conveniência & 4 \\
\hline Lavras, 2011 & $\begin{array}{l}\text { Analisar o papel da APS nas Redes Regionais de } \\
\text { Atenção à Saúde, que se organizam no SUS na } \\
\text { perspectiva de superar a fragmentação sistêmica } \\
\text { existente, apontando iniciativas voltadas a seu } \\
\text { aprimoramento }\end{array}$ & $\begin{array}{l}\text { Revisão da } \\
\text { literatura }\end{array}$ & Não se aplica & 4 \\
\hline Silva, 2011 & $\begin{array}{l}\text { Analisar os desafios presentes no Sistema Único } \\
\text { de Saúde para promover uma melhor integração } \\
\text { entre os serviços e organizar redes }\end{array}$ & $\begin{array}{l}\text { Revisão da } \\
\text { literatura }\end{array}$ & Não se aplica & 4 \\
\hline $\begin{array}{c}\text { Motta et al., } \\
2011\end{array}$ & $\begin{array}{l}\text { Investigar o conhecimento e a percepção dos } \\
\text { profissionais das equipes, e problematizar as } \\
\text { dificuldades apontadas na atuação junto aos } \\
\text { idosos, discutindo o papel da ESF na rede de } \\
\text { atenção ao idoso no Brasil hoje }\end{array}$ & $\begin{array}{c}\text { Estudo } \\
\text { qualitativo }\end{array}$ & Conveniência & - \\
\hline $\begin{array}{c}\text { Campos et al., } \\
2011\end{array}$ & $\begin{array}{l}\text { Avaliar a articulação entre as redes de atenção } \\
\text { primária e de saúde mental em regiões de alta } \\
\text { vulnerabilidade social de uma grande cidade } \\
\text { brasileira (Campinas - SP) através dos } \\
\text { parâmetros: pesquisa avaliativa, participativa e } \\
\text { predominantemente qualitativa }\end{array}$ & $\begin{array}{c}\text { Estudo } \\
\text { qualitativo }\end{array}$ & Conveniência & - \\
\hline Mendes, 2010 & Discutir a importância da conformação das RAS & $\begin{array}{l}\text { Revisão da } \\
\text { literatura }\end{array}$ & Não se aplica & 4 \\
\hline
\end{tabular}


Da amostra, 94,5\% (17) dos trabalhos foram publicados na área da saúde em geral e 5,5\% (1) especificamente na área de Enfermagem. No que se refere ao ano de publicação, notou-se que
$44,4 \%$ (8) dos estudos eram de 2011, 22,2\% (4) de 2010, 11,1\% (2) de 2009, 6,0\% (1) de 2008, 11,1 (2) de 2007 e $6,0 \%$ (1) de 2006 . Não foi localizado estudo anterior a 2006.

Quadro 1. continuação

\begin{tabular}{|c|c|c|c|c|}
\hline Referência & Objetivo & $\begin{array}{l}\text { Tipo de } \\
\text { estudo }\end{array}$ & $\begin{array}{c}\text { Plano } \\
\text { amostral }\end{array}$ & $\begin{array}{l}\text { Nível de } \\
\text { evidência }\end{array}$ \\
\hline $\begin{array}{l}\text { Báscolo e } \\
\text { Yavich, } 2010\end{array}$ & $\begin{array}{l}\text { Descrever o desenvolvimento da } \\
\text { política APS promovida pelo Município } \\
\text { de Rosário }\end{array}$ & $\begin{array}{l}\text { Estudo de } \\
\text { caso }\end{array}$ & Não menciona & 4 \\
\hline $\begin{array}{l}\text { Pessôa et al., } \\
2010\end{array}$ & $\begin{array}{l}\text { Relatar experiências das redes de atenção ao } \\
\text { idoso no Brasil }\end{array}$ & $\begin{array}{l}\text { Estudo de } \\
\quad \text { caso }\end{array}$ & Conveniência & 4 \\
\hline $\begin{array}{c}\text { Kuschnir e } \\
\text { Chorny, } 2010\end{array}$ & $\begin{array}{l}\text { Buscar referências na literatura e na experiência } \\
\text { internacional que possam contribuir para o } \\
\text { debate da constituição de redes no SUS }\end{array}$ & $\begin{array}{l}\text { Revisão da } \\
\text { literatura }\end{array}$ & Não se aplica & 4 \\
\hline $\begin{array}{l}\text { Almeida et al., } \\
2010\end{array}$ & $\begin{array}{l}\text { Analisar o desenvolvimento de estratégias e } \\
\text { instrumentos de coordenação desde a Estratégia } \\
\text { Saúde da Família (ESF) aos demais níveis do } \\
\text { sistema de saúde com foco no conjunto de } \\
\text { medidas pró-coordenação vinculado à } \\
\text { "integração entre níveis assistenciais" }\end{array}$ & $\begin{array}{l}\text { Estudo de } \\
\text { caso }\end{array}$ & Conveniência & 4 \\
\hline $\begin{array}{l}\text { Giovanella } \\
\text { et al., } 2009\end{array}$ & $\begin{array}{l}\text { Analisar a implementação da ESF com foco na } \\
\text { integração e atuação intersetorial em quatro } \\
\text { capitais para discutir as potencialidades da ESF } \\
\text { como estratégia de APS abrangente }\end{array}$ & $\begin{array}{l}\text { Estudo de } \\
\text { caso }\end{array}$ & Conveniência & 4 \\
\hline $\begin{array}{l}\text { Lima e Rivera, } \\
2009\end{array}$ & $\begin{array}{l}\text { Discutir uma abordagem teórico-metodológica } \\
\text { para estudos na área da integração de serviços de } \\
\text { saúde, destacando o aspecto intersubjetivo dos } \\
\text { processos de coordenação }\end{array}$ & $\begin{array}{l}\text { Revisão da } \\
\text { literatura }\end{array}$ & Não se aplica & 4 \\
\hline $\begin{array}{c}\text { Roese e } \\
\text { Gerhardt, } 2008\end{array}$ & $\begin{array}{l}\text { Construir um perfil socioeconômico e } \\
\text { demográfico dos usuários, de forma a ilustrar a } \\
\text { utilização de dois serviços de saúde de média } \\
\text { complexidade, por meio do mapeamento dos } \\
\text { fluxos de usuários de municípios da Metade Sul } \\
\text { do Estado do Rio Grande do Sul }\end{array}$ & $\begin{array}{l}\text { Estudo } \\
\text { descritivo, } \\
\text { transversal }\end{array}$ & Conveniência & 4 \\
\hline $\begin{array}{l}\text { Costa-e-Silva } \\
\text { et al., } 2007\end{array}$ & $\begin{array}{l}\text { Descrever uma experiência de integração entre } \\
\text { serviços de saúde }\end{array}$ & $\begin{array}{l}\text { Estudo de } \\
\text { caso }\end{array}$ & Conveniência & 4 \\
\hline $\begin{array}{c}\text { Vázquez et al., } \\
2007\end{array}$ & $\begin{array}{l}\text { Identificar os componentes relacionais de uma } \\
\text { rede internacional de organizações, cooperação } \\
\text { técnica e financeira para promover o } \\
\text { desenvolvimento de sistemas de saúde baseados } \\
\text { nos cuidados de saúde primários nos países da } \\
\text { região das Américas }\end{array}$ & $\begin{array}{c}\text { Estudo } \\
\text { transversal }\end{array}$ & Conveniência & 4 \\
\hline $\begin{array}{l}\text { Giovanella, } \\
2006\end{array}$ & $\begin{array}{l}\text { Investigar as configurações institucionais do } \\
\text { primeiro nível de atenção à saúde nos países da } \\
\text { União Europeia }\end{array}$ & $\begin{array}{l}\text { Revisão da } \\
\text { literatura }\end{array}$ & Não se aplica & 4 \\
\hline
\end{tabular}


No tocante ao delineamento metodológico, percebeu-se que 39,1\% (7) dos estudos utilizaram a revisão de literatura, 16,6\% (3) apresentaram abordagem qualitativa, 5,5\% (1) abordagem quantitativa, $11,1 \%$ (2) abordagem mista (qualitativa e quantitativa), 16,6\% (3) estudo de caso e 11,1\% (2) relato de experiência. Os artigos com abordagem metodológica mista não mencionaram os testes estatístico realizados. Já o artigo quantitativo utilizou o teste estatístico quiquadrado, com probabilidade do Erro tipo I menor ou igual a $0,05(\mathrm{p} \leq 0,05)$.

Quanto ao plano amostral definido para os estudos, 55,5\% (10) consistia em amostragem por conveniência, 5,5\% (1) não mencionava o plano amostral e os outros 39,0\% (7) dos artigos não exigiam amostragem por constituírem estudos de revisão. No que concernem aos critérios considerados para composição da amostra, 50,0\% (9) apresentam os critérios para arrolar os sujeitos na pesquisa, no entanto, $11,1 \%$ (2) não explicitam e em 38,9\% (7) o planejamento amostral não se aplicava. Em relação às limitações e possíveis vieses, notou-se que 100\% (18) dos manuscritos não apresentavam tais informações.

Quanto à classificação da hierarquia do nível de evidência científica dos manuscritos, 88,9\% (16) foram classificados quanto ao nível de evidência 4, e 11,1\% (2) dos artigos, pela natureza qualitativa , não foi possível a categorização, haja vista que o instrumento utilizado não previa este tipo de estudo.

No que tange às fragilidades, às potencialidades, aos desafios e às perspectivas da APS na coordenação das RAS, cujos resultados encontrados estão listados no Quadro 2, observou-se que alguns trabalhos trazem como desafios a realização de estudos voltados às APS e às RAS.

\section{Discussão}

Observou-se a relevância do objeto investigado para o cenário nacional e também para a ciência, haja vista o impacto destas investigações para o fortalecimento do SUS e por disparar novos horizontes e linhas investigativas. O número de artigos sobre o tema vem crescendo, o que reflete um investimento nacional no tocante à revisão dos sistemas de saúde, cujas expectativas estão devotadas à busca de soluções para as condições crônicas ${ }^{24}$.

Os resultados demonstram que as condições crônicas exigem um sistema de atenção que responda de forma proativa, contínua e integrada, por meio do compromisso político com a APS, à implementação de um acolhimento qualificado e das práticas inovadoras no âmbito da política e da atenção ${ }^{25}$. Contudo, a APS como eixo coordenador ainda encontra vários desafios, sobretudo pela fragilidade de se implantar uma rede que permita a integração entre os níveis de atenção na sua dimensão vertical e horizontal, bem como sistemas de apoio e logística para sustentar os fluxos de comunicação e os processos para a produção social em saúde.

Dentre os trabalhos contemplados na revisão, notou-se que eles foram publicados por diferentes áreas da saúde, demonstrando a característica multidisciplinar da temática. Desses estudos, observou-se que nenhum foi anterior ao ano de 2006, o que pode estar relacionado às políticas de saúde voltadas ao fortalecimento e indução da APS, à expansão da ESF e aos incentivos financeiros federais ${ }^{26-28}$.

Não foi possível observar a categoria profissional que mais publicou sobre a APS na coordenação de redes, possivelmente porque se trata de um tema de investigação inerente às profissões, de caráter universal.

Outro aspecto analisado se refere ao nível de evidência científica, em que a maioria dos artigos foi classificada como fraca, pois seu delineamento metodológico pautou-se em revisões e estudos de caso. Embora, os achados desses estudos não pudessem ser generalizados para outras realidades e contextos, trazem lições importantes acerca do fortalecimento dos sistemas de saúde no tocante ao modelo de proteção social inspirado na APS.

Parece haver, nos estudos, um consenso sobre a necessidade de novas investigações com nível significativo na escala hierárquica de classificação das evidências científicas, através de revisões sistemáticas.

Cabe ressaltar, que a consistência epistemológica e o rigor metodológico são considerados requisitos de qualidade e impacto das pesquisas. Na presente investigação, a ênfase emerge, em sua maioria, no procedimento de coleta de dados, em que apenas metade dos estudos incluídos na pesquisa, descrevia os critérios de inclusão. Outro achado importante é que, em muitos dos manuscritos, o plano amostral considerado não estava apropriado à natureza do estudo.

Quanto à questão norteadora, pôde-se observar que fragilidades, potencialidades e perspectivas/desafios relacionados à APS como coordenadora das RAS são abordados de forma convergente nos estudos. Sobre as fragilidades, verificou-se como nó crítico a coordenação das 
Quadro 2. Principais fragilidades, potencialidades, perspectivas/desafios, encontrados nos estudos, relacionados à Atenção Primária à Saúde como coordenadora das Redes de Atenção à Saúde.

\begin{tabular}{|c|c|c|}
\hline Fragilidades & Potencialidades & Perspectivas/Desafios \\
\hline $\begin{array}{l}\text {. Dificuldade de coordenação } \\
\text { entre os níveis de atenção; } \\
\text {. Precário funcionamento do } \\
\text { mecanismo de referência e } \\
\text { contra referência; } \\
\text {. Desconhecimento dos vários } \\
\text { pontos de atenção pelos } \\
\text { profissionais de APS; } \\
\text { - Baixo financiamento do SUS; } \\
\text {. Dificuldade de cooperação } \\
\text { entre profissionais de atenção } \\
\text { primária e secundária; } \\
\text { - Descompromisso dos } \\
\text { trabalhadores com os resultados } \\
\text { organizacionais; } \\
\text {. Acolhimento incipiente na } \\
\text { Estratégia Saúde da Família } \\
\text { (ESF); } \\
\text {. Práticas de promoção da saúde } \\
\text { não consolidadas; } \\
\text {. Deficiência nos processos } \\
\text { comunicacionais; } \\
\text {. Descompasso entre o Plano } \\
\text { Diretor Regionalização - PDR e } \\
\text { a prática dos usuários; } \\
\text {. Dificuldade de acesso à } \\
\text { atenção especializada. }\end{array}$ & $\begin{array}{l}\text {. Maior envolvimento do Agente } \\
\text { Comunitário de Saúde; } \\
\text {. Expansão da cobertura da ESF; } \\
\text {. Institucionalização de novas } \\
\text { práticas na atenção voltada para a } \\
\text { integração assistencial (programas } \\
\text { de educação permanente, } \\
\text { subsistema integrado materno } \\
\text { infantil); } \\
\text {. Implantação de centrais } \\
\text { informatizadas de regulação e } \\
\text { marcação de procedimentos; } \\
\text {. Implantação de protocolos } \\
\text { clínicos; } \\
\text { - Melhoria dos resultados clínicos e } \\
\text { diminuição dos custos da } \\
\text { assistência em sistema de saúde } \\
\text { organizados em RAS; } \\
\text {. Aumento da oferta de APS com } \\
\text { diminuição das barreiras de acesso; } \\
\text {. Estruturação da APS como porta } \\
\text { de entrada do sistema; } \\
\text {. Ampliação da resolubilidade da } \\
\text { APS; } \\
\text { - Articulação em ações de vigilância } \\
\text { e atenção; } \\
\text { - Avanços na integração da ESF à } \\
\text { rede de atenção à saúde; } \\
\text { - Investimento em tecnologia de } \\
\text { informação e comunicação, com } \\
\text { implantação de sistemas } \\
\text { informatizados de regulação e } \\
\text { prontuários eletrônicos. }\end{array}$ & $\begin{array}{l}\text {. Vontade política para maiores } \\
\text { investimentos na APS } \\
\text { (investimento em estrutura física, } \\
\text { capacitação de pessoal, fluxo e } \\
\text { processo de trabalho, etc.); } \\
\text {. Rompimento das características } \\
\text { hegemônica do modelo de } \\
\text { atenção atual; } \\
\text {. Profissionais comprometidos } \\
\text { com um processo de trabalho } \\
\text { horizontal, em que se combine } \\
\text { especialização com } \\
\text { interdisciplinaridade; } \\
\text {. Fortalecimento da ESF como } \\
\text { porta de entrada no sistema de } \\
\text { saúde; } \\
\text {. Referências reguladas pela ESF } \\
\text { para atenção especializada; } \\
\text {. Fortalecimento do papel do } \\
\text { generalista na condução dos } \\
\text { cuidados; } \\
\text {. Incentivos adequados para } \\
\text { generalistas; } \\
\text {. Usuários e especialistas abertos } \\
\text { aos novos modelos } \\
\text { organizacionais; } \\
\text {. Implantação de novas práticas } \\
\text { baseadas no agir comunicativo; } \\
\text {. Engajamento político em prol } \\
\text { da regionalização; } \\
\text {. Maior divulgação da ESF; } \\
\text {. Realização de estudos voltados à } \\
\text { APS e RAS. }\end{array}$ \\
\hline
\end{tabular}

redes pela APS circunstancial à carência de mecanismos e estratégias de integração e comunicação, de regulação do acesso pelos diferentes níveis, e a ausência de sistemas informatizados que possibilitem a gestão destes processos e fluxos ${ }^{3,29}$.

A integração em sistemas de serviços de saúde depende da qualidade dos processos de coordenação e estes, por sua vez, dependem da eficácia da comunicação instituída entre as pessoas no interior do sistema. Estudos apontam que as falhas na coordenação de serviços organizados em forma de redes podem ser decorrentes das deficiências dos processos comunicacionais e, portanto, novas práticas baseadas no agir comunicativo são de grande valia. $\mathrm{O}$ agir comuni- cativo apresenta um caminho para a transformação da prática cotidiana, no qual se evoluiria de uma perspectiva autoritária, fragmentada e individualista para uma visão democrática, integrada, baseada no trabalho coletivo, na solidariedade e na comunicação ${ }^{30}$.

Experiências de três municípios brasileiros em relação à Estratégia Saúde da Família (ESF) e à atenção ao idoso revelaram o desconhecimento dos profissionais da APS acerca dos vários pontos de atenção da rede, o que além de comprometer a resolubilidade da atenção primária, produz desgastes neles, que solitariamente por vezes se incumbem de contornar os problemas de um sistema de saúde fragmentado ${ }^{29}$. 
Para que a APS seja considerada coordenadora das redes de atenção à saúde tornam-se necessários investimentos em tecnologias nas unidades de saúde, adequação da infraestrutura física, introdução de sistemas logísticos e de apoio e também a construção de novas unidades de saúde, dentre outros ${ }^{31}$.

Na revisão, pôde-se constatar que as potencialidades da APS se sobrepõem às fragilidades, destacando-se os avanços na integração da ESF à rede assistencial, o aumento da oferta desta modalidade de APS, a institucionalização de novas práticas na atenção voltada para a integração assistencial (programas de educação permanente), a implantação de protocolos clínicos, dentre outros. Em algumas localidades, onde fica patente o compromisso político com a APS, temse transgredido da posição de porta de entrada para a de coordenadora, instaurando-se a corresponsabilidade pela saúde dos usuários. Os resultados nestes cenários se mostram bem significativos pela resolubilidade, integralidade e equidade ${ }^{32}$.

As experiências mencionadas em Florianópolis, Belo Horizonte e Vitória revelam mecanismos para a integração da APS aos outros pontos de atenção, como instituição da Política de formação de recursos humanos, centrais de regulação informatizadas agregadas à APS e estabelecimento de diretrizes de fluxos e introdução dos protocolos clínicos. Ademais, há que mencionar que a expansão da cobertura da ESF atrelada a uma política permanente de formação de recursos humanos tem garantido a articulação/integração para um trabalho em rede ${ }^{3,33}$.

Apesar de resultados importantes para a instituição de uma política em prol da APS, ainda há desafios, com destaque para o comprometimento político com a APS e o estabelecimento de fluxos para acesso aos serviços de saúde, como apontam estudos de avaliação da atenção aos idosos e à saúde mental ${ }^{34-36}$. Em pesquisa desenvolvida por Silva ${ }^{37}$, com objetivo de analisar os desafios do SUS na promoção de uma efetiva integração entre os serviços de saúde e de conformá-los em redes de atenção, constatou-se que a coordenação pela APS depende de um projeto político amplo e ofensivo, que englobe todos setores de um sistema de proteção social e a própria sociedade civil.

Outro desafio importante refere-se à ruptura paradigmática, da superação do modelo voltado às condições agudas e de busca por cuidados face à agudização de uma condição crôni$\mathrm{ca}^{24}$, por um modelo de cuidados mais dialógico com produção de sentidos para quem cuida e também para quem é cuidado. A ampliação da clínica do profissional da APS é um dispositivo importante para suscitar reflexões e novos arranjos para uma APS com efeito de coordenar. $\mathrm{Na}$ Alemanha e em outros países europeus, há um grande investimento no médico generalista para a clínica e, assim, para a continuidade dos cuidados. Em sete dos 15 países da União Europeia (Dinamarca, Itália, Portugal, Espanha, Reino Unido, Irlanda e Holanda), o generalista exerce a função de "gatekeeper", ou seja, este tem poder sobre os outros níveis de atenção ${ }^{31,38,39}$.

Do exposto, os desafios a serem vencidos para garantir a APS como coordenadora das RAS são diversos. Todavia, os trabalhos realizados indicam as potencialidades a serem implementadas em uma perspectiva abrangente de APS, condicionada às adaptações do modelo de saúde vigente e à ampliação dos recursos profissionais, assistenciais, tecnológicos e de infraestrutura.

As limitações encontradas para a realização da presente revisão se referiram ao uso das palavras-chave e descritores no SciELO para localização dos artigos na respectiva base de dados eletrônica, o que pode incorrer na ausência de algum estudo que tenha sido publicado abordando a temática investigada. Observou-se uma variedade de métodos empregados para a avaliação da APS na conformação das redes, o que impede uma análise comparativa mais refinada dos resultados alcançados. Mesmo nos estudos transversais selecionados, a amostragem foi por conveniência, agregando assim outros vieses, além de memória e de causalidade reversa. Como toda pesquisa, a revisão apresenta vantagens e limitações potenciais na sua aplicação, merecendo o cuidado necessário, especialmente no que concerne às conclusões dos achados e, assim, na prevenção de prováveis equívocos subjetivos. Sobre a potencialidade do trabalho, os autores destacam a metodologia lançada, que foi validada por importantes expertises na cátedra de revisão.

\section{Conclusão}

Os resultados indicam que a organização dos sistemas de saúde em redes de atenção à saúde coordenadas pela APS pode desempenhar um impacto significativo na saúde da comunidade, com custos suportáveis, estando engendrada no arcabouço jurídico e político do SUS.

A partir desta revisão pode-se concluir sobre a necessidade de delineamentos metodológicos 
mais robustos, com consistência interna e validade externa, com vistas à apresentação à comunidade científica e geral das evidências científicas da capacidade da APS coordenar uma RAS.

Dos trabalhos selecionados, nenhum estudo se conformou com forte evidência científica, todavia trouxeram reflexões acerca dos dilemas locais para a instituição de um sistema coordenado pela APS. Nas referidas localidades vem ocorrendo reformas institucionais e políticas importantes para o fortalecimento da APS.

Vale considerar que o objeto pleiteado nesta revisão é relativamente novo e que muitos dos estudos realizados, os estudos de caso e qualitativos, vêm cumprindo o seu papel de descrever novas realidades e difundir boas práticas de gestão no SUS no que se refere à coordenação dos cuidados e conformação de redes. Esta opção epistemológica é justificada no próprio interesse do trabalho de buscar situações singulares e particulares da APS, que apesar de apresentarem infirma evidência, eles, como os estudos controlados, são igualmente relevantes para a saúde coletiva.

Espera-se que as indagações erigidas no presente estudo possam fomentar novas investigações, com substantiva validade e possibilidades de generalizações, subsidiando desta forma as políticas de saúde vigente e a organização dos sistemas de saúde em redes, coordenado por uma APS ampliada.

\section{Colaboradores}

LBB Rodrigues coordenou o grupo de trabalho, elaborou o texto-base, realizou o levantamento bibliográfico, a sistematização, a análise e a discussão dos artigos, e editou o texto final. PCS Silva auxiliou no levantamento bibliográfico, participou da sistematização, análise e discussão dos artigos. RC Peruhype colaborou com a redação. PF Palha realizou a revisão crítica. MP Popolin e JA Crispim colabororaram com a redação. IC Pinto e AA Monroe realizaram a revisão crítica. RA Arcêncio realizou a revisão crítica e aprovou a versão final do manuscrito.

\section{Referências}

1. World Health Organization (WHO). Noncommunicable diseases country profiles 2011. Geneva: WHO; 2011.

2. Giovanella L, Mendonça MHM, Almeida PF, Escorel S, Senna MCM, Fausto MCR, Delgado MM, Andrade CLT, Cunha MS, Martins MIC, Teixeira CP. Saúde da família: limites e possibilidades para um abordagem integral de atenção primária à saúde no Brasil. Cien Saude Colet 2009; 14(3): 783-794.

3. Almeida PF, Giovanella L, Mendonça MHM, Escorel S. Desafios à coordenação dos cuidados em saúde: estratégias de integração entre níveis assistenciais em grandes centros urbanos. Cad Saude Publica 2010; 26(2):286-298.

4. Boerma WGW. Coordination and integration in European primary care. In: Saltman RS, Rico A, Boerma WGW, editors. Primary care in the driver's seat? Organizational reform in European primary care. Berkshire: Open University Press; 2007. p. 3-21.

5. De Maeseneer J, Willems S, De Sutter A, Van de Geuchte I, Billings M. A atenção primária à saúde como estratégia para alcançar a equidade de assistência à saúde: uma revisão da literatura, iniciativa da rede de conhecimento em sistemas de saúde. Rev Brasileira Saúde da Família 2008; Ano IX(19):46-72.

6. World Health Organization (WHO). Primary health care. Report of the international conference on primary health care, Alma-Ata. Geneva: WHO; 1978.

7. Almeida PF, Fausto MCR, Giovanella L. Fortalecimento da atenção primária à saúde: estratégia para potencializar a coordenação dos cuidados. Rev. Panam Salud Publica 2011; 29(2):84-95.

8. Haggerty JL, Yavich N, Báscolo EP, Grupo de Consenso sobre un Marco de Evaluación de la Atención Primaria en América Latina. Un marco de evaluación de la atención primaria de salud en América Latina. Rev Panam Salud Publica 2009; 26(5):377-384. 
9. Gomes KO, Cotta RMM, Araújo RMA, Cherchiglia ML, Martins TCP. Atenção primária à saúde - a "menina dos olhos" do SUS: sobre as representações sociais dos protagonistas do sistema único de saúde. Cien Saude Colet 2011; 16(Supl. 1):881-892.

10. Mendes EV. A atenção primária à saúde no SUS. Fortaleza: Escola de Saúde Pública do Ceará; 2002.

11. Conill EM, Fausto MCR, Giovanella L. Contribuições da análise comparada para um marco abrangente na avaliação de sistemas orientados pela atenção primária na América Latina. Rev. Bras. Saude Matern. Infant 2010; 10(Supl. 1):15-27.

12. Organização Pan-Americana da Saúde (OPAS). A atenção à saúde coordenada pela APS: construindo as redes de atenção no SUS: contribuições para o debate. Brasília: OPAS; 2011.

13. Mendes EV. As redes de atenção à saúde. Brasília: OPAS; 2011.

14. Brasil. Portaria $n^{\circ} 4279$ de 30 de dezembro de 2010 . Estabelece diretrizes para a organização da rede de atenção á saúde no âmbito do sistema único de saúde (SUS). Diário Oficial da União 2010; $30 \mathrm{dez}$.

15. Kringos DS, Boerma WGW, Hutchinson A, Zee J, Groenewegen PP. The breadth of primary care: a systematic literature review of its core dimensions. BMC Health Services Research 2010; 10:65.

16. Nnaji GA. Shifting Focus of Primary Health Care in the Context of Health Systems Reform in Resource Poor Settings. Primary Health Care: Open Access 2011; 1(1):1-5.

17. Cooper HM. The Integrative research review. Beverly Hills: SAGE Publications; 1984.

18. Ganong LH. Integrative reviews of nursing research. Res Nurs Health 1987; 10(1):1-11.

19. Broome ME. Integrative literature reviews in the development in nursing: foundations, techniques and applications. Philadelphia: W. B. Saunders Company; 1993.

20. Ursi ES. Prevenção de lesões de pele no perioperatório: revisão integrativa da literatura [dissertação]. Ribeirão Preto: Universidade de São Paulo; 2005.

21. Polit DF, Beck CT, Hungler BP. Fundamentos de pesquisa em enfermagem: métodos, avaliação e utilização. 5a Edição. Porto Alegre: Artmed; 2004.

22. Dawson AP. Asthma in the Australian Indigenous population: a review of the evidence. Rural and Remote Health 2004; 4(1):238.

23. Sackett DL, Straus SE, Richardson WS, Rosenberg W, Haynes RB. Evidence-based medicine - How to pratice and teach EMB. $2^{\text {nd }}$ Edition. Oxford: Churchill Livingstone; 2000.

24. Mendes EV. As redes de atenção à saúde. Cien Saude Colet 2010; 15(5):2297-2305.

25. Mendes EV. O Cuidado das condições crônicas na Atenção Primária à Saúde: o imperativo da consolidação da Estratégia de Saúde da Família. Brasília: Organização Pan-americana de Saúde (OPAS); 2012.

26. Brasil. Portaria no 399/GM, de 22 de fevereiro de 2006. Divulga o Pacto pela Saúde 2006 - Consolidação do SUS e aprova as Diretrizes Operacionais do Referido Pacto. Diário Oficial da União 2006; 22 fev.
27. Brasil. Portaria n 648/GM, de 28 de março de 2006. Aprova a Política Nacional de Atenção Básica, estabelecendo a revisão de diretrizes e normas para a organização da Atenção Básica para o Programa Saúde da Família (PSF) e o Programa Agentes Comunitários de Saúde (PACS). Diário Oficial da União 2006; 28 mar.

28. Brasil. Portaria $\mathrm{n}^{\circ} 1.654$, de 19 de julho de 2011. Institui, no âmbito do Sistema Único de Saúde, o Programa Nacional de Melhoria do Acesso e da Qualidade da Atenção Básica (PMAQ-AB) e o Incentivo Financeiro do PMAQ-AB, denominado Componente de Qualidade do Piso de Atenção Básica Variável - PAB Variável. Diário Oficial da União 2011; 19 jul.

29. Motta LB, Aguiar AC, Caldas CP. Estratégia Saúde da Família e a atenção ao idoso: experiências em três municípios brasileiros. Cad Saude Publica 2011; 27(4):779-786.

30. Lima JC, Rivera FJU. Agir comunicativo, redes de conversação e coordenação em serviços de saúde: uma perspectiva teórico-metodológica. Comunicação Saúde Educação 2009; 31(12):329-342.

31. Lavras C. Atenção Primária à Saúde e a Organização de Redes Regionais de Atenção à Saúde no Brasil. Saude Soc 2011; 20(4):867-874.

32. Giovanella L. A atenção primária à saúde nos países da União Européia: configurações e reformas organizacionais na década de 1990. Cad Saude Publica 2006; 22(5):951-963.

33. Costa-e-Silva V, Rivera FJ, Hortale VA. Projeto Integrar: avaliação da implantação de serviços integrados de saúde no Município de Vitória, Espírito Santo, Brasil. Cad Saude Publica 2007; 23(6):1405-1414.

34. Roese A, Gerhardt TE. Fluxos e utilização de serviços de saúde: mobilidade dos usuários de média complexidade. Rev Gaucha Enferm 2008; 29(2):221229.

35. Pessôa LR, Ferasso M, Vargas LM, Ferla AA. Challenges in organizing care networks for the elderly in two regions of Brazil. Cad Saude Publica 2010; 26(7):1314-1322.

36. Campos RO, Gama CA, Ferrer AL, Santos DVD, Stefanello S, Trapé TL, Porto K. Saúde mental na atenção primária à saúde: estudo avaliativo em uma grande cidade brasileira. Cien Saude Colet 2011; 16(12):4643-4652.

37. Silva SF. Organização de redes regionalizadas e integradas de atenção à saúde: desafios do Sistema Único de Saúde (Brasil). Cien Saude Colet 2011; 16(6):2753-2762.

38. Kuschinir R, Chorny AH. Redes de atenção à saúde: contextualizando o debate. Cien Saude Colet 2010; 15(5):2307-2316.

39. Giovanella L. Redes integradas, programas de gestão clínica e generalista coordenador: análise das reformas recentes do setor ambulatorial na Alemanha. Cien Saude Colet 2011; 16(Supl. 1):1081-1096.

Artigo apresentado em 08/08/2012

Aprovado em 27/09/2012

Versão final apresentada em 01/10/2012 